\title{
Trigeminal Neuralgia
}

National Institute of Neurological Disorders and Stroke (NINDS)

\section{Source}

National Institute of Neurological Disorders and Stroke (NINDS). Trigeminal Neuralgia

Information Page.

Trigeminal neuralgia (TN), also called tic douloureux, is a chronic pain condition that causes extreme, sporadic, sudden burning or shock-like face pain. The pain seldom lasts more than a few seconds or a minute or two per episode. The intensity of pain can be physically and mentally incapacitating. TN pain is typically felt on one side of the jaw or cheek. Episodes can last for days, weeks, or months at a time and then disappear for months or years. In the days before an episode begins, some patients may experience a ting ling or numbing sensation or a somewhat constant and aching pain. The attacks often worsen over time, with fewer and shorter pain-free periods before they recur. The intense flashes of pain can be triggered by vibration or contact with the cheek (such as when shaving, washing the face, or applying makeup), brushing teeth, eating, drinking, talking, or being exposed to the wind. TN occurs most often in people over age 50, but it can occur at any age, and is more common in women than in men. There is some evidence that the disorder runs in families, perhaps because of an inherited pattern of blood vessel formation. Although sometimes debilitating, the disorder is not lifethreatening. The presumed cause of TN is a blood vessel pressing on the trigeminal nerve in the head as it exits the brainstem. TN may be part of the normal aging process but in some cases it is the associated with another disorder, such as multiple sclerosis or other disorders characterized by damage to the myelin sheath that covers certain nerves. 